\title{
Perfil fitoquímico e ensaio microbiológico dos extratos da entrecasca de Maytenus rigida Mart. (Celastraceae)
}

\author{
Charles S. Estevam, ${ }^{*}, 1,2$ Amaro M. Cavalcanti, ${ }^{1}$ Érica Verena F. Cambui, ${ }^{2}$ Vítor Araújo \\ Neto, ${ }^{2}$ Paulo T. G. Leopoldo, ${ }^{2}$ Roberta P. M. Fernandes, ${ }^{2}$ Brancilene S. de Araujo, ${ }^{2}$ \\ Zenaldo Porfírio, ${ }^{3}$ Antonio Euzébio G. Sant'Ana ${ }^{1}$
}

\author{
${ }^{1}$ Laboratório de Pesquisa em Recursos Naturais, Universidade Federal de Alagoas, Tabulerio dos Martins s/n, \\ 57000-000 Maceió-AL, Brasil, \\ ${ }^{2 *}$ Laboratório de Bioquímica, Universidade Federal de Sergipe, Av. Marechal Rondom s/n, Rosa Elze, 49100-000 \\ São Cristóvão-SE, Brasil, \\ ${ }^{3}$ Laboratório de Microbiologia, Centro de Ciências Biológicas , Universidade Federal de Alagoas, Tabulerio dos \\ Martins s/n, 57000-000, Maceió-AL, Brasil
}

\begin{abstract}
RESUMO: Maytenus rigida Mart (Celastraceae), conhecida por “Bom-homem”, “Bom-nome”, “Cabelo-de-negro", “Casca-grossa” e "Pau-de-colher”, é uma arvore de pequeno porte. A entrecasca do caule é empregada popularmente no Nordeste do Brasil no tratamento das dores em geral, infecções e inflamações. O presente trabalho avaliou tanto o perfil fitoquímico de $M$. rigida por meio de um roteiro analítico, quanto à atividade antibiótica dos extratos pelo método de Kirby-Bauer modificado. Os resultados demonstraram que os extratos etanólico, aquoso, clorofórmico, acetato de etila e hidroalcoólico de $M$. rigida apresentam atividade antibacteriana contra Escherichia coli, Pseudomonas aeruginosa e Staphylococcus aureus, enquanto que a fração hexânica não exibe qualquer atividade. Catequinas, quinonas, esteróides, triterpenos, saponinas, flavonóides e compostos fenólicos foram detectados na análise fitoquímica.
\end{abstract}

Unitermos: Maytenus rigida, entrecasca do caule, atividade antimicrobiana, perfil fitoquímico.

\begin{abstract}
Phytochemistry and microbiological assay of the bark extracts of Maytenus rigida Mart. (Celastraceae)”. Maynetus rigida Mart (Celastraceae), known as "Bom-homem”, "Bom-nome”, "Cabelo-de-negro", "Casca-grossa" and "Pau-de-colher”, is a small tree. The stem bark is used by the population in the northeast of Brazil to treat aches, infections and inflammations in general. The present work evaluated both the phytochemistry of $M$. rigida Mart by an analytical routine, and the antimicrobial activity of the bark extracts by the KirbyBauer modified method. Our results showed the aqueous, methanol, chloroform, ethyl acetate and hydroalcoolic extracts of M. rigida Mart has antimicrobial activity against Escherichia coli, Pseudomonas aeruginosa and Staphylococcus aureus, while the hexane extract does not have any activity. Catechins, quinones, steroids, triterpenes, saponins, flavonoids and phenolic compounds were detected by the phytochemical analysis.
\end{abstract}

Keywords: Maytenus rigida, bark, antimicrobial activity, phytochemical analysis.

\section{INTRODUÇÃO}

O desenvolvimento da resistência de microrganismos a antibióticos correntemente em uso tem sido rápido e progressivo. Este problema, associado à baixa taxa de descoberta de novas substâncias com potencial antimicrobiano, é causa de preocupação. Além do linezolide (Zyvox®), a última classe de antibióticos que chegou ao mercado farmacêutico dos EUA foi há 30 anos (White et al., 2003). Uma das medidas utilizadas na batalha contra a resistência bacteriana é a modificação dos antibióticos em uso; no entanto, pouco sucesso tem sido obtido. Por esses motivos, a descoberta de novas moléculas, de fonte natural ou sintética, com atividade antimicrobiana é assunto de máxima urgência. A diversidade de moléculas encontradas em plantas faz das mesmas promissoras fontes de novos agentes antimicrobianos (Leitão et al., 2006; Silva et al., 2007, 2008; Coutinho et al., 2008).

Maytenus rigida faz parte da família Celastracea que compreende cerca de 85 gêneros. Essa família é composta por aproximadamente 900 espécies de ampla distribuição nas regiões tropicais e subtropicais de todo o mundo (Barroso, 1991; Brummit, 1992; Joly, 1993). O gênero Maytenus é formado por espécies arbustivas ou arbóreas encontradas em matas e campos (Barroso, 
1991; Joly, 1993) que são largamente utilizadas pelos povos da América do Sul. As plantas desse gênero são ricas em metabólitos bioativos da classe dos terpenos ou isoprenóides (Shirota et al., 1994; Niero et al., 2006; Reyes et al., 2006; Gutieerrez et al., 2007) e atividades biológicas tais como antiinflamatória (Sanogo et al., 2006; Reyes et al., 2006; Santos et al., 2007; Sosa et al., 2007), antiulcerogênica (Mota et al., 2008), antimalaria (Muregi et al., 2007; Muthaura et al., 2007), antioxidante (Brunni et al., 2006; Buffa et al., 2004; Vellosa et al., 2006), antinoceptiva (Niero et al., 2006; Sanogo et al., 2006), antitumoral (Shirota et al., 1994; Buffa et al., 2004) e anticonvulsivante (Quintans-Júnior et al., 2008) já foram relatadas para diversas espécies desse gênero.

A espécie do gênero Maytenus rigida, conhecida como "Bom-homem", "Bom-nome", "Cabelo-de-negro", "Casca-grossa” e "Pau-de-colher”, é uma arvore de pequeno porte, sendo que a entrecasca do caule apresenta aspectos terapêuticos, sendo empregada nos casos de dores em geral, infecções e inflamações (Mota \& Albuquerque, 2002; Agra et al., $2007,2008)$. Apesar do uso medicinal dessa planta no tratamento de infecções, não existem relatos de testes antimicrobianos usando essa espécie. Este trabalho relata o perfil fitoquímico e atividade antimicrobiana in vitro dos extratos aquoso e etanólico da entrecasca de Maytenus rigida.

\section{MATERIAL E MÉTODOS}

\section{Material vegetal}

A entrecasca da planta de Maytenus rigida Mart. (Celastraceae), foi coletada fora do período da floração, no povoado Capim Grosso - AL, Brasil. A planta foi identificada pelo Dr. Carlos Dias da Silva Júnior do Departamento de Biologia da Universidade Federal de Sergipe. Um espécime foi registrado no herbário da Universidade Federal de Sergipe, número 00767. As amostras foram colocadas em estufa (Marconi modelo MA-037) a $37^{\circ} \mathrm{C}$, com renovação e circulação de ar por $48 \mathrm{~h}$ até completa desidratação.

\section{Preparação dos extratos}

\section{Preparação do extrato alcoólico}

Para o preparo do extrato etanólico foram pesados 1381,4 g do pó das entrecascas que foram misturados com etanol comercial 90\% em percolador de aço inoxidável durante cinco dias. Após concentração do solvente em evaporador rotativo sob pressão reduzida, o extrato etanólico bruto da entrecasca $(561,9$ g) foi obtido. Este extrato etanólico foi então suspenso em uma solução $\mathrm{MeOH} / \mathrm{H}_{2} \mathrm{O}$ (metanol-água, 2:3) e extraído exaustiva e sucessivamente em $n-\mathrm{C}_{6} \mathrm{H}_{14}$ (hexano), $\mathrm{CHCl}_{3}$ (clorofórmio) e AcOEt (acetato de etila). Os extratos alcoólicos e suas fases obtidas após a partição foram concentrados em evaporador rotatório (Büchi modelo RE-114B) até total remoção do solvente e submetidos a ensaios fitoquímicos e microbiológico in vitro. Todos os solventes usados para a extração, exceto o etanol, foram da marca VETEC.

Para o ensaio microbiológico, $200 \mathrm{mg}$ do extrato bruto foram solubilizados em $1 \mathrm{~mL}$ de $\mathrm{MeOH}: \mathrm{H}_{2} \mathrm{O}$ 1:3. Dessa solução, uma alíquota de $40 \mu \mathrm{L}$ foi utilizada. Todos os experimentos foram realizados utilizando-se como controle $\mathrm{MeOH}: \mathrm{H}_{2} \mathrm{O}$ 1:3.

\section{Preparação do extrato aquoso}

O pó das entrecascas (450 g), após secagem à temperatura ambiente e trituração em moinho mecânico, foi submetido à extração por decocção com $900 \mathrm{~mL}$ de água destilada. Após extração, o material foi filtrado à vácuo e liofilizado.

Esse extrato foi submetido aos mesmos ensaios microbiológicos que o extrato alcoólico, onde $200 \mathrm{mg}$ do mesmo foram solubilizados em $\mathrm{MeOH}: \mathrm{H}_{2} \mathrm{O}$ conforme descrito previamente.

\section{Prospecção fitoquímica}

$\mathrm{Na}$ prospecção fitoquímica, a ocorrência de alcalóides, antocianidinas, antocianinas, ácidos fixos, aurona, catequinas, chalconas, esteróides, flavonóis, flavonóides, fenóis, heterosídeos cianogênicos, leucoantocianidinas, quinonas, resinas, saponinas, taninos e xantonas foi avaliada de acordo com o método proposto por Wagner \& Bladt (1995) e Matos (1997).

\section{Ensaio antimicrobiano}

\section{Microrganismos}

Os isolados bacterianos Staphylococcus aureus (BAC -94), S. aureus (BAC -97), S. aureus (BAC -115), Escherichia coli (BAC -148), Pseudomonas aeruginosa (BAC -104), P. aeruginosa (BAC -135) e Salmonela typhi (BAC -155), bem como os isolados de leveduras do gênero Candida, a saber, Candida albicans (BAC -01), C. kruse (BAC -09) e C. tropicalis (BAC -15) utilizados nos ensaios microbiológicos, foram doadas pelo Laboratório de Patologia Clínica da Santa Casa de Misericórdia de Maceió.

\section{Manutenção dos microrganismos}

Os microrganismos foram mantidos em estufa bacteriológica (Marconi modelo MA-037) a $37{ }^{\circ} \mathrm{C}$ por $48 \mathrm{~h}$ em meio de cultura BHI, caldo nutriente, bacto-ágar e ágar Mueller-Hinton, que foram preparados de acordo com as instruções do fabricante (DIFCO) e esterilizados em autoclave a $121^{\circ} \mathrm{C}$ durante 15 minutos. 
Tabela 1. Prospecção fitoquímica dos extratos de entrecasca de Maytenus rigida.

\begin{tabular}{|c|c|c|c|c|c|c|}
\hline \multirow[t]{2}{*}{ Constituintes Químicos } & \multicolumn{6}{|c|}{ Extratos } \\
\hline & Etanólico & Aquoso & Hexano & Clorofórmio & $\begin{array}{l}\text { Acetato de } \\
\text { etila }\end{array}$ & Hidroalcoólico \\
\hline Ácidos fixos & - & - & - & - & - & - \\
\hline Alcalóides & - & - & - & - & - & - \\
\hline Antocianidina & - & - & - & - & - & - \\
\hline Antocianinas & - & - & - & - & - & - \\
\hline Auronas & - & - & - & - & - & - \\
\hline Catequinas & + & + & - & + & + & + \\
\hline Chalconas & - & - & - & - & - & - \\
\hline Esteróides & + & + & + & + & - & - \\
\hline Fenóis & + & + & - & + & + & + \\
\hline Flavonóis & + & + & - & + & + & + \\
\hline Flavononóis & + & + & - & + & + & + \\
\hline Flavonóides & + & + & - & + & + & + \\
\hline $\begin{array}{l}\text { Heterosídeos } \\
\text { Cianogênicos }\end{array}$ & - & - & - & - & - & - \\
\hline Leucoantocianidinas & + & + & - & + & + & + \\
\hline Quinonas & + & + & - & + & + & + \\
\hline Resinas & - & - & - & - & - & - \\
\hline Saponinas & + & + & - & + & + & + \\
\hline Taninos & + & - & - & - & - & - \\
\hline Triterpenos & + & + & + & + & - & - \\
\hline Xantonas & + & - & - & - & - & - \\
\hline
\end{tabular}

Tabela 2. Atividade antimicrobiana do extrato etanólico bruto da entrecasca de Maytenus rigida*.

\begin{tabular}{lc}
\hline Microrganismo & Zona de inibição (mm) \\
\hline Candida albicans $(01)$ & - \\
Candida kruse (09) & - \\
Candida tropicalis (15) & - \\
Staphylococcus aureus $(97)$ & 12 \\
Pseudomonas aeruginosa (135) & - \\
Escherichia coli $(148)$ & 14 \\
\hline
\end{tabular}

*são considerados ativos os extratos que formaram zonas de inibição $\geq 10 \mathrm{~mm}$.

- não apresentou atividade na concentração de 200 mg/mL.

Testes de sensibilidade e resistência das linhagens microbianas aos extratos

Para avaliar a sensibilidade e resistência dos microrganismos aos extratos de $M$. rigida foi utilizado o método de Kirby-Bauer modificado (difusão em ágar). Poços com capacidade para $50 \mu \mathrm{L}$ de solução foram produzidos em placas de Petri pela introdução de ponteiras plásticas na superfície de vidro antes da adição do meio de cultura ágar-ágar. Em seguida, uma alíquota da solução do microrganismo a ser testado foi ressuspendida em meio ágar Mueller-Hinton (concentração final de $1 \times 10^{3}$ unidades formadoras de colônia $/ \mathrm{mL}$ ) a $45^{\circ} \mathrm{C}$. Esta solução foi então vertida na placa de Petri contendo o meio solidificado e após 10 minutos as ponteiras foram retiradas. Em cada poço resultante foram colocados $40 \mu \mathrm{L}$ dos extratos de $M$. rigida, sendo que o poço central foi usado como controle (água-metanol 33\%). As placas foram mantidas em estufa bacteriológica aquecida a $37^{\circ} \mathrm{C}$, por 24 horas, e a atividade antimicrobiana foi determinada de acordo com a medida do halo de inibição do crescimento em volta do poço com auxílio de halômetro. Os extratos foram considerados ativos quando apresentaram halos de inibição superiores a $10 \mathrm{~mm}$.

\section{RESULTADOS E DISCUSSÃO}

Plantas do gênero Maytenus são ricas em terpenos da classe dos tripernos (Shirota et al., 1994; Niero et al., 2006; Reyes et al., 2006; Gutieerrez et al., 2007), embora também tenham sido encontrados flavonóides (Sannomiya et al., 1998; Xie et al., 2007; Tiberti et al., 2007), glicolipídios (Mendes et al., 2006) e alcalóides (Corsino et al., 1998). O perfil fitoquímico da entrecasca de $M$. rigida (Tabela 1 ) revelou a presença de triterpenos, mas também de outras classes de substâncias: catequinas, quinonas, esteróides, saponinas, 
Tabela 3. Atividade antibacteriana das frações obtidas por partição do extrato bruto etanólico e extrato aquoso da entrecasca de Maytenus rigida*.

\begin{tabular}{|c|c|c|}
\hline Extrato & Bactéria & Zona de inibição (mm) \\
\hline \multirow[t]{4}{*}{ Aquoso } & Staphylococcus aureus (94), (97) e (115) & 15,16 е 16 \\
\hline & Escherichia coli $(148)$ & 16 \\
\hline & Pseudomonas aeruginosa (104) e (135) & 16 e 25 \\
\hline & Salmonela typhi (155) & - \\
\hline \multirow{4}{*}{ Hexânico } & Staphylococcus aureus (94), (97) e (115) & - \\
\hline & Escherichia coli $(148)$ & - \\
\hline & Pseudomonas aeruginosa (104) e (135) & - \\
\hline & Salmonela typhi (155) & - \\
\hline \multirow{4}{*}{$\mathrm{CHCl}_{3}$} & Staphylococcus aureus (94), (97) e (115) & 11,11 e 11 \\
\hline & Escherichia coli (148) & 13 \\
\hline & Pseudomonas aeruginosa (104) e (135) & 14 e 21 \\
\hline & Salmonela typhi (155) & - \\
\hline \multirow[t]{5}{*}{ AcOEt } & Staphylococcus aureus (94), (97) e (115) & 15,16 e 16 \\
\hline & Escherichia coli (148) & 14 \\
\hline & Pseudomonas aeruginosa (104) e (135) & 16 e 21 \\
\hline & Salmonela typhi (155) & - \\
\hline & Staphylococcus aureus (94), (97) e (115) & 12,13 e 13 \\
\hline \multirow[t]{3}{*}{$\mathrm{H}_{2} \mathrm{O} / \mathrm{MeOH}$} & Escherichia coli $(148)$ & 14 \\
\hline & Pseudomonas aeruginosa (104) e (135) & 13 e 22 \\
\hline & Salmonela typhi (155) & - \\
\hline
\end{tabular}

*são considerados ativos os extratos que formaram zonas de inibição $\geq 10 \mathrm{~mm}$.

- não apresentou atividade na concentração de 200 mg/mL.

flavonóides e compostos fenólicos. Entretanto, não foram detectadas substâncias da classe dos alcalóides, taninos, heterosídeos, resinas, chalconas, ácidos fixos e leucoantocianidinas.

O extrato etanólico bruto da entrecasca (Tabela 2) apresentou atividade antimicrobiana tanto para a bacteria Gram positiva $S$. aureus (BAC -97) quanto para a Gram negativa $E$. coli (BAC -148), não sendo observada qualquer atividade contra as leveduras $C$. albicans (BAC -01), C. kruse (BAC -09) e C. tropicalis (BAC -15). Com base nesses resultados, o extrato etanólico foi submetido à partição liquído-liquído objetivando a obtenção de frações enriquecidas para o biomonitoramento da atividade antimicrobiana.

$\mathrm{O}$ extrato hexânico $\left(\mathrm{C}_{6} \mathrm{H}_{14}\right)$ não apresentou atividade antibacterina. Os extratos clorofórmico $\left(\mathrm{CHCl}_{3}\right)$, acetato de etila (AcOEt), hidroalcoólico $\left(\mathrm{H}_{2} \mathrm{O} /\right.$ $\mathrm{MeOH})$ e aquoso liofilizado apresentaram atividade frente as cepas bactérianas $S$. aureus (BAC -94), $S$. aureus (BAC -97), S. aureus (BAC -115), E. coli (BAC -148), P. aeruginosa (BAC -104) e P. aeruginosa (BAC -135). O extrato etanólico bruto, que anteriormente não apresentava atividade antimicrobiana contra a bactéria $P$. aeruginosa (BAC -135), apresentou, após fracionamento, atividade positiva para todas as frações obtidas, excetuando-se a hexânica (Tabela 3). Esse resultado demonstra a importância de um fracionamento preliminar dos extratos para a deteç̧ão da atividade antimicrobiana, uma vez que a baixa concentração dos compostos ativos podem mascarar a deteç̧ão nos extratos brutos. Nenhuma das frações obtidas da partição do extrato etanólico causou a inibição do crescimento da bactéria S. typhi (BAC -155).

Entre as substâncias detectadas na análise fitoquímica dos extratos, os flavonóides (Xie et al., 2007), terpenos (González et al., 1996; Wang et al., 2007) e saponinas (Avato et al., 2006) já foram reportados anteriormente por possuírem atividade antimicrobiana. A presença desses compostos nos extratos de $M$. rigida pode explicar a atividade antibacteriana encontrada.

Em conclusão, demostramos que a planta Maytenus rigida é uma potencial fonte de agentes antibacterianos, o que motiva posteriores estudos para o isolamento e identificação dos principios ativos responsáveis por essa atividade que podem ser usadas na indústria farmacêutica.

\section{AGRADECIMENTOS}

Os autores agradecem a Dr. Carlos Dias da Silva Júnior pela identificação e caracterização da espécie vegetal. Este trabalho foi financiado pela Fundação de Amparo a Pesquisa do Estado de Alagoas (FAPEAL) e pelo Conselho Nacional de Desenvolvimento Científico e Tecnológico (CNPq).

\section{REFERENCIAS}

Agra MF, França PF, Barbosa-Filho JM 2007. Synopsis of the plants known as medicinal and poisonous in Northeast of Brazil. Rev Bras Farmacogn 17: 114140.

Agra MF, Silva KN, Basílio IJLD, França PF, Barbosa-Filho 
JM 2008. Survey of medicinal plants used in the region Northeast of Brazil. Rev Bras Farmacogn 18: 472-508.

Avato P, Bucci R, Tava A, Vitali C, Rosato A, Bialy Z, Jurzysta M 2006. Antimicrobial activity of saponins from Medicago sp. Structure-activity relationship. Phytother Res 20: 454-457.

Barroso GM 1991. Sistemática de Angiospermas do Brasil. Viçosa: Imprensa Universitária.

Brummitt RK 1992. Vascular Plant Families and Genera. Royal Botanic Gardens: Kew.

Bruni R, Rossi D, Muzzoli M, Romagnoli C, Paganetto G, Besco E, Choquecillo F, Peralta K, Lora WS, Sacchetti G 2006. Antimutagenic, antioxidant and antimicrobial properties of Maytenus krukovii bark. Fitoterapia 77: 538-545.

Buffa W, Bolzani VD, Furlan M, Pereira SIV, Pereira AMS, Franca SC 2004. In vitro propagation of Maytenus ilicifolia (Celastraceae) as potential source for antitumoral and antioxidant quinomethide triterpenes production. A rapid quantitative method for their analysis by reverse-phase high-performance liquid chromatography. Arkivoc 6: 137-146.

Corsino J, Bolzani VS, Pereira AMS, França SC, Burlan M 1998. Bioactive sesquiterpene pyridine alkaloids from Maytenus aquifolium. Phytochemistry 48: 137140.

Coutinho HDM, Costa JGM, Siqueira-Júnior JP, Lima EO 2008. In vitro anti-staphylococcal activity of Hyptis martiusii Benth against methicillin-resistant Staphylococcus aureus-MRSA strains. Rev Bras Farmacogn 18 (Supl.): 670-675.

González AG, Alvarenga NL, Ravelo AG, Jimenez IA, Bazzocchi IL, Canela NJ, Moujir LM 1996. Antibiotic phenol nor-triterpenes from Maytenus canariensis. Phytochemistry 43: 129-132.

Gutiérrez F, Estévez-Braun A, Ravelo AG, Astudillo L, Zarate R 2007. Terpenoids from the medicinal plant Maytenus ilicifolia. J Nat Prod 70: 1049-1052.

Joly AB 1993. Botânica - Introdução à Taxonomia Vegetal. São Paulo: Companhia Editora Nacional.

Leitão SG, Castro O, Fonseca EM, Julião LS, Tavares ES, Leo RRT, Vieira RC, Oliveira DR, Leitão GG, Martino V, Sulsen V, Barbosa YAG, Pinheiro DPG, Silva PEA, Teixeira DF, Lourenço MCS 2006. Screening of Central and South American plant extracts for antimycobacterial activity by the Alamar Blue test. Rev Bras Farmacogn 16: 6-11.

Matos FJA 1997. Introdução à Fitoquímica Experimental. Fortaleza: Edições UFC.

Mendes BG, Machado MJ, Falkenberg M 2006. Triagem de glicolipídios em plantas medicinais. Rev Bras Farmacogn 16: 568-575.

Mota CN, Albuquerque UP 2002. As Muitas fases da Jurema: De espécie botânica à divindade afro-indígena. Rio de Janeiro. Recife: Edições Bagaço.

Mota KSL, Pita JCLR, Estevam EC, Medeiros VM, Tavares JF, Agra MF, Diniz MFFM, Silva MS, Batista LM 2008. Evaluation of the toxicity and antiulcerogenic activity of the ethanol extract of Maytenus obtusifolia Mart. Leaves. Rev Bras Farmacogn 18: 441-446.

Muthaura CN, Rukunga GM, Chhabra SC, Omar SA, Guantai AN, Gathirwa JW, Tolo FM, Mwitari PG, Keter LK, Kirira PG, Kimani CW, Mungai GM, Njagi EN 2007. Antimalarial activity of some plants traditionally used in treatment of malaria in Kwale District of Kenya. J Ethnopharmacol 112: 545-551.

Muregi FW, Ishih A, Miyase T, Suzuki T, Kino H, Amano T, Mkoji GM, Terada M 2007. Antimalarial activity of methanolic extracts from plants used in Kenyan ethnomedicine and their interactions with chloroquine (CQ) against a CQ-tolerant rodent parasite, in mice. J Ethnopharmacol 111: 190-195.

Niero R, Mafra AP, Lenzi AC, Cechinel-Filho V, Tischer CA, Malheiros A, De Souza MM, Yunes RA, Delle Monache F 2006. A new triterpene with antinociceptive activity from Maytenus robusta. Nat Prod Res 20: 1315-1320.

Quintans-Júnior LJ, Almeida JRGS, Lima JT, Nunes XP, Siqueira JS, Oliveira LEG, Almeida RN, AthaydeFilho PF, Barbosa-Filho JM 2008. Plants with anticonvulsant properties - a review. Rev Bras Farmacogn 18 (Supl.): 798-819.

Reyes CP, Núñez MJ, Jiménez IA, Busserolles J, Alcaraz MJ, Bazzocchi IL 2006. Activity of lupane triterpenoids from Maytenus species as inhibitors of nitric oxide and prostaglandin E2. Bioorg Med Chem 14: 15731579.

Sannomiya M, Vilegas W, Rastrelli L, Pizza C 1998. A Flavonoid glycoside from Maytenus aquifolium. Phytochemistry 49: 237-239.

Sanogo R, Diallo D, Maiga A, De Tommasi N, De Pasquale R 2006. Analgesic and anti-inflammatory activities of the aqueous extracts of Maytenus senegalensis, Stereospermum kunthianum and Trichilia emetica used in the treatment of dysmenorrhoea in Mali. Planta Med 72: 1059-1059.

Santos VL, Costa VBM, Agra MF, Silva BA, Batista LM 2007. Pharmacological studies of ethanolic extracts of Maytenus rigida Mart (Celastraceae) in animal models. Rev Bras Farmacogn 17: 336-342.

Shirota O, Morita H, Takeya K, Itokawa H 1994. Cytotoxic aromatic triterpenes from Maytenus ilicifolia and Maytenus chuchuhuasca J Nat Prod 57: 1675-1681.

Silva JG, Souza IA, Higino JS, Siqueira-Junior JP, Pereira JV, Pereira MSV 2007. Atividade antimicrobiana do extrato de Anacardium occidentale Linn. em amostras multiresistentes de Staphylococcus aureus. Rev Bras Farmacogn 17: 572-577.

Silva MAR, Higino JS, Pereira JV, Siqueira-Júnior JP, Pereira MSV 2008. Antibiotic activity of the extract of Punica granatum Linn. over bovine strains of Staphylococcus aureus. Rev Bras Farmacogn 18: 209-212.

Sosa S, Morelli CF, Tubaro A, Cairoli P, Speranza G, Manitto P 2007. Anti-inflammatory activity of Maytenus senegalensis root extracts and of maytenoic acid. Phytomedicine 14: 109-114.

Tiberti LA, Yariwake JH, Ndjoko K, Hostettmann K 2007. Identification of flavonols in leaves of Maytenus ilicifolia and M. aquifolium (Celastraceae) by LC/ UV/MS analysis. J Chromatogr B 846: 378-384.

Vellosa JCR, Khalil NM, Formenton VAF, Ximenes VF, Fonseca LM, Furlan M, Brunetti IL, Oliveira OMMF 2006. Antioxidant activity of Maytenus ilicifolia root bark. Fitoterapia 77: 243-244.

Wagner H, Bladt S 1995. Plant Drug Analysis - A Thin Layer Chromatography Atlas. Berlin: Springer.

Wang YF, Wang XY, Lai GF, Lu CH, Luo SD 2007. Three new sesquiterpenoids from the aerial parts of Homalomena occulta. Chem Biodivers 4: 925-931.

White RJ, Margolis PS, Trias J, Yuan Z 2003. Targeting metalloenzymes: a strategy that works. Curr Opin Pharmacol 3: 502-507.

Xie J, Sun W, Duan K, Zhang Y 2007. Chemical constituents of roots of Epimedium wushanense and evaluation of their biological activities. Nat Prod Rep 21: 600-605. 